\title{
A Research on Coding Information Diffusion on Geographical Space in IOI for Disaster Mitigation
}

\author{
Yiran Yin ${ }^{1,2,3}$, Chongfu Huang ${ }^{1,2,3, *}$ \\ ${ }^{1}$ Key Laboratory of Environmental Change and Natural Disaster, Ministry of Education, Beijing Normal \\ University, Beijing 100875, China \\ ${ }^{2}$ State Key Laboratory of Earth Surface Processes and Resource Ecology, Beijing Normal University, Beijing \\ 100875, China \\ ${ }^{3}$ Faculty of Geographical Science, Academy of Disaster Reduction and Emergency Management, Beijing \\ Normal University, Beijing 100875, China
}

\section{救灾智联网中地理空间信息扩散的编程研究}

\author{
尹怡然 ${ }^{1,2,3}$, 黄崇福 ${ }^{1,2,3, *}$ \\ ${ }^{1}$ 北京师范大学, 环境演变与自然灾害教育部重点实验室, 北京 100875 , 中国 \\ ${ }^{2}$ 北京师范大学, 地表过程与资源生态国家重点实验室, 北京 100875 , 中国 \\ ${ }^{3}$ 北京师范大学, 地理科学学部, 减灾与应急管理研究院, 北京 100875 , 中国
}

\begin{abstract}
In order to apply the technology of information diffusion on geographical space in internet of intelligences (IOI) for disaster mitigation, which plays an important role in multi-level precision rescue for severe natural disasters, this paper reviews the main programming technologies in the existing IOI and proposes a technological path for IOI for disaster mitigation. The advanced path includes managing background database with MySQL, updating the disaster damage information by means of network server clock, and calculating automatically by running the PHP code. The method above enables us to update the disaster damage data of appointed geographic units by inter-unit information diffusion. Taking GWR as an example, this paper gives the basic framework for programming information diffusion on geographical space in IOI for disaster mitigation.
\end{abstract}

基金项目: 国家重点研发计划 (编号: 2017YFC1502902), 国家自然科学基金项目（编号：41671502）。

*通讯作者: hchongfu@bnu.edu.cn
Keywords: Internet of intelligences, demands of assistance, geography unit, information diffusion

摘要

为使重大自然灾害多层级精准救助中地理 空间信息扩散这一关键技术能在救灾智联网中 实现, 本文回顾了现有智联网中的主要编程技术, 提出了用 MySQL 管理背景数据库, 依服务器时钟 扫描灾区数据更新状态, 用 PHP 程序实现将已知 地理单元上获得的灾情信息, 向没有获得灾情信 息的地理单元进行扩散, 从而填补不完备数据的 技术路径。本文以地理回归模型为例, 给出了在 救灾智联网中编程实现地理空间信息扩散的基 本框架。

关键词: 智联网; 救助需求; 地理单元; 信息扩 散

1. 引言

救助需求是指在救灾过程中灾区对物资、人 员和救灾设备等的需求。在救灾过程中尤其是应 急阶段, 可支配物资、人员和设备的数量是有限 的, 需要基于灾区各行政单位的灾情信息进行应 急救助决策, 把有限的资源进行合理的调配, 实 现自然灾害精准救助。应急救助方案的制定是救 
灾决策的关键一环, 直接关乎因灾死亡人数与经济损 失的多少。然而在我国, 灾情信息主要通过上报与调 研的方式进行统计, 需要一定的时间周期。且自然灾 害尤其是重大自然灾害发生后, 交通、通讯中断, 在 灾后数小时内灾情信息难以及时获取与更新。灾情信 息的不完备严重制约着灾害救助的时效性与精准性。 为此急需研发自然灾害灾情快速评估平台, 为灾害管 理人员提供有效的决策支撑。

灾情信息的不完备一是由于数据收集上的滞后, 二是由于灾情本身尚在发展, 无法获知最终结果。因 此自然灾害灾情快速评估平台的目标是基于现有信 息, 实现灾区特定行政单元的现时灾情快速评估。为 此, 一方面需要选取合适的数学方法基于不完备数据 进行灾情评估, 另一方面需要选取合适的工具研发自 然灾害灾情快速评估系统, 实现灾情评估的在线计算 和结果共享。

信息扩散技术能够充分挖掘不完备数据中的有 效信息, 已从数学上被证明能有效处理小样本问题 ${ }^{[1]}$, 在自然灾害风险分析等领域得到了广泛的应用 ${ }^{[2]}$ 。因 此可以基于信息扩散原理构建预测模型对不完备数 据进行计算。但已有的信息扩散技术是在概率空间内 的扩散, 而灾情数据通常是以各级行政单位为基本单 位来进行统计的, 这意味着由已知信息预测灾情数据 是在地理空间上的扩散。文 [3] 提出了地理空间上信 息扩散的理念, 对这一概念给出了形式化定义。以背 景数据为媒介, 地理空间上信息扩散能将不完整数据 补充为完整数据, 为实现灾情快速评估的算法原理指 明了前景。

智联网 (Internet of intelligences, IOI) 一开始是 作为一种风险分析在线服务方法被黄崇福提出的 ${ }^{[4]}$, 最简单的智联网由图 1 所示。现已有包括高考志愿填 报智联网服务平台、智联网驱动的风险雷达、海洋环

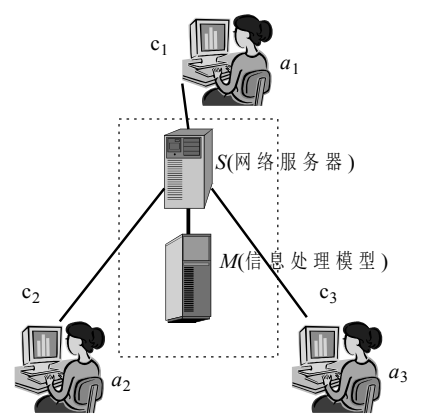

图 1. 最简单的智联网。三台计算机形成网络, 三个工作 人员是三个智能体。
境风险管理智联网平台、风险时效性评价等在内 的 10 个智联网平台面世。智联网的应用已不仅 限于风险分析, 而是作为一个生产智慧产品的网 络被逐渐应用于科研、生产、生活等各个领域 ${ }^{[5]}$ 。 现有智联网平台已有了一套比较成熟的技术框 架: 使用PHP 语言、基于 Yii 框架进行系统研发, 使用 MySQL 数据库进行数据管理。孙雁南利用开 源前端库 Leaflet 首次将智联网与 WebGIS 技术 相结合, 研发了具有地理信息系统支撑的智联网 [6], 这为智联网在地理、自然灾害相关领域的应 用奠定了基础。

为构建 “救灾智联网”, 本文以地理空间上 的信息扩散技术为信息处理模型, 实现灾情快速 评估, 并将灾情信息通过在线地理信息系统进行 共享。要实现救灾智联网需突破以下三个关键技 术: 一是设计服务于救灾智联网的核心功能的数 据库; 二是实现数据库定时扫描和任务触发; 三 是实现后台在线计算。本文探讨如何形成这些技 术的途径。

\section{2. 数据库系统设计与数据管理}

MySQL 是一个基于客户机/服务器

(Client/Server, C/S) 的关系型数据库管理系 统, 具有开源、性能高效、跨平台支持、支持多 用户等特点, 是中小型网站开发的首选。可以使 用 PHP 语言编写 SQL 原生语句实现对 MySQL 数据 库进行数据查找、增加、删除、更新、导出、恢 复等操作。Yi 框架作为一个基于组件、用于开 发大型Web 应用的高性能 PHP 框架, 提供了 $\mathrm{db}$ 组件来访问和操作存储在数据库中的数据,

与 SQL 原生语句相比更具直观性和灵活性, 简化 了管理批量数据的步骤, 提高了数据库管理的效 率。基于 Yii 框架研发救灾智联网, 使用户在网 页上即可实现上述所有功能, 以高度可视化的方 式进行数据库的管理而无需登录数据库后台。

救灾智联网数据库包括背景数据、灾情数 据、用户信息三个主体, 对应设计三张数据表, 如图 2 所示。救灾智联网的用户为灾害管理人员, 用户信息表包括编号、姓名、学历、工作单位等 字段, 用于记录其基本身份信息, 同时据此划分 用户等级, 对应享有不同的数据管理权限。背景 数据表和灾情数据表均以研究区行政单元为单 位进行记录, 前者用于记录各行政单元的自然地 理属性和政治经济属性, 后者用于记录灾害发生 后各行政单元的灾情信息。背景数据表的完善需 要在实际应用前完成, 属于数据库中的基础数据, 


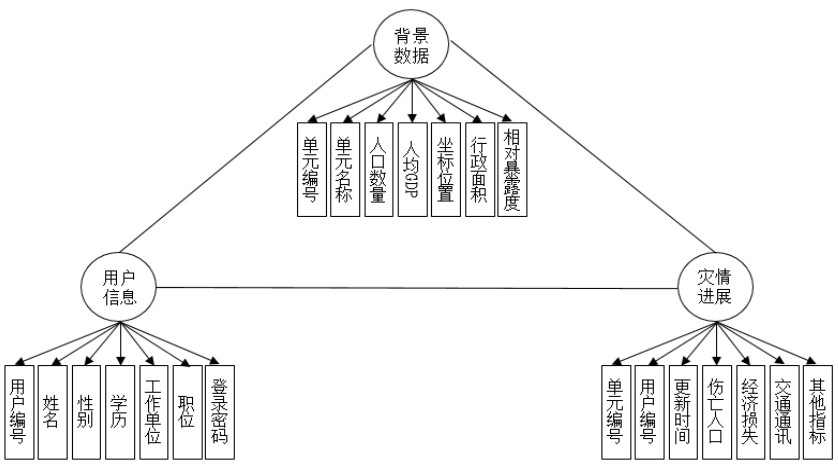

图 2. 救灾智联网数据库结构

可依据统计年鉴等进行日常维护。灾情数据表中的各 字段是随着灾害发展而动态变化的, 在救灾期间由灾 害管理人员进行实时更新, 同时也作为快速评估的输 出值以弥补统计途径所得数据的不完备。

\section{3. 救灾智联网的定时数据更新}

为确保灾情快速评估平台的时效性, 为地理空间 信息扩散计算模块添加网络计时器, 将灾情快速评估 延伸为定时任务, 同时便于及时跟踪灾情发展。在 PHP 语言中获取当前时间十分便捷, 通过以下语句即 可实现

data (format, timestamp);

其中参数 format 表示可以使用不同的方式来格式化 日期和时间, 参数 timestamp 可选, 默认将时间转换 为整数表示的时间唯。如

echo date("Y-m-d H:i:s");

输出的结果为

2018-01-01 01:01:01

此外需要注意, date()获取的是服务器时间, 若要获 取研究区时间需要使用函数 date_default_timezone _set()或 ini_set()来设置时区。如

ini_set ('date.timezone','Asia/Beijing');

表示设置为北京时间。

在 Yii 框架中创建定时任务可以通过调用控制台 命令 (ConsoleCommands) 来实现, 分成下述两个关 键步骤。首先在工程文件包的控制台模块中新建 PHP 文件（以 test.php 为例）, 定义命名空间, 创建 Controller 并继承 yiilconsolelController, 后编写灾情 评估计算函数。代码整体结构如下:

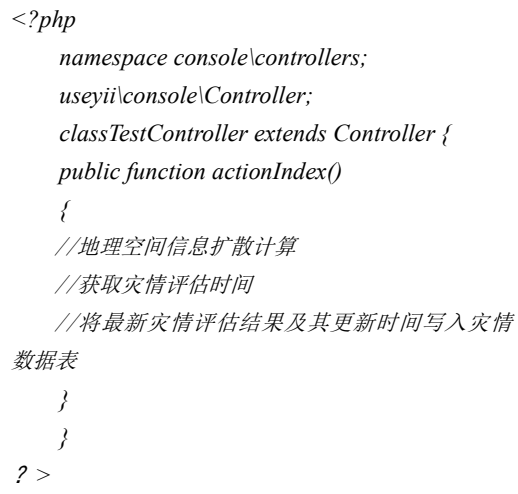

随后在命令提示符中使用 yii 命令测试该 文件是否可以成功运行。若可以, 再运行 crontab-e 命令来设置定时任务执行上述 PHP 文 件, 代码示例如下

$0 * 1 * * * /$ usr/local/php/your_project_path/test.php 其中前 6 位表示将时间间隔设置为 1 小时且每天 执行, 其后语句表示执行上述 PHP 文件。

这样, 通过上述两个步骤就实现了全天整点 灾情自动评估及数据库的更新。

\section{4. 地理空间信息扩散的 PHP 编程原理}

Yii 框架采用模块 - 视图 - 控制器 (Mode1-View-Controller, MVC) 的基本模式, 具有清晰的设计框架 ${ }^{[7]}$ 其中模型代表数据、业务 逻辑和规则, 视图展示模型的输出, 控制器接受 出入并将其转换为模型和视图命令。采用 $\mathrm{Yii}$ 框架来搭建救灾智联网, 借助其 gi 模块快速生 成模型、视图和控制器模板, 不需要重新编写业 
务逻辑, 从而可以极大地压缩开发周期。在救灾智联 网平台中嵌入灾情快速评估模块, 充分发挥 MVC 模式 逻辑清晰、的特点, 将任务合理分配至三个模块中配 合实现。

如文 [3] 所述地理空间信息扩散模型的原理, 其输 入为各行政单元的背景数据, 如人口数量、人均 GDP 等, 在减灾智联网系统中这些数据储存在数据库的背 景数据表中; 输出为经济损失、死亡人数等灾情数据, 储存在灾情数据表中。出于定时任务功能实现的路径 要求和系统代码的简明性考虑, 如上节所述, 模型计 算代码单独编写进 PHP 文件并归于控制器模块, 实现 与数据库的交互, 即数据读取和更新。

完成数据库更新后, 下一步是将灾情评估结果借 助在线地理信息系统进行展示, 此处则需要借助视图 模块进行实现。将信息扩散时间及结果以列表方式和 地图方式显示在救灾智联网平台页面上。

\section{5. 实现地理空间信息扩散的基本框架}

用于找寻具有空间分布特征的两个或多个变量 之间数量关系的地理加权回归模型（Geographical Weighted Regression, GWR），其实是一种线性假设 条件下的地理空间信息扩散模型, 式 (1) 为其表达 式 $^{[8]}$ 。

$$
y_{i}=a_{0}+\sum_{k=1, m} a_{k} x_{i k}+\varepsilon_{i}
$$

与传统全局回归模型不同, 式中回归参数 $a_{k}$ 随空间 样本点 $\left(u_{i}, v_{i}\right)$ 的不同而不同, 即其为地理空间位置 的函数, 从而可以有效揭示数据的空间非平稳性。

地理加权回归建立了空间不均匀的预测模型, 可 以粗略地表达研究区背景数据和灾情数据间的量化 关系, 然而本质是线性回归, 很难充分表达非线性地 表的现象。但据此模型确实可以在研究变量和解释变 量建立一个明确的数学关系, 因此可将其视为一个最 简单的地理空间信息扩散模型。我们以此模型为例, 集成上述三节的内容, 形成地理空间信息扩散的基本 框架。

灾情可以用许多指标来衡量, 比如伤亡人数、经 济损失等, 针对不同的灾种也有更为细致的指标体系, 如描述地震灾情常用烈度等指标, 描述洪水灾情常用 淹没范围、积水深度、淹没时间等指标。以上灾情指 标值构成了制定灾害救助方案的重要参考依据, 减灾 智联网的数据库也依据以上指标而设计（图 2)。当 某一强度的灾害发生后, 不同地区的灾情受当地承灾 体脆弱性的制约而有所不同, 同时随着时间的推移在 发生变化, 救灾智联网旨在通过地理空间信息扩散这
一数学模型, 一方面展示各受灾单元的灾情差异, 同时及时跟进灾情的动态变化。

灾情可以视为是灾害强度和承灾体脆弱性的 综合效应, 例如某单元经济损失 $L$ 可以被视作是 其人口数量 $\left(\omega_{1}\right)$ 、人均 GDP $\left(\omega_{2}\right)$ 、相对暴 露度 $\left(\omega_{3}\right)$ 等变量的函数。以地理加权回归模型 为例, 此函数关系可以用下式来表达:

$$
L_{i}=a_{0}+\sum_{k=1,3} a_{k} \omega_{i k}+\varepsilon_{i}
$$

通过某一灾害的历史数据可以计算出解释 变量对应的回归系数 $a_{k}\left(u_{i}, v_{i}\right)$, 从而建立起区 域性的灾情预测模型, 并将其作为背景数据库的 一部分, 用于实际灾害发生时的灾情评估 (图 3)。

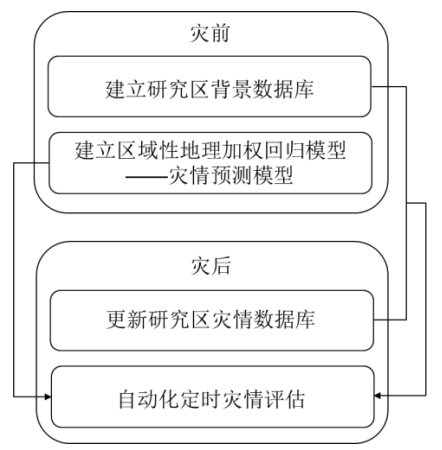

图 3. 救灾智联网地理空间信息扩散实现基本框架

\section{6. 地理加权回归模型在救灾智联网中的初步实} 现

在已知研究区地理加权回归模型的基础上 实现在线评估功能, 需要三步: 在灾情数据库中 读取数据、代入模型进行计算、将灾情评估结果 录入数据库。代码如下:

foreach (Disaster::find()->batch(10) as

\$disasters) \{

$\$ y=0$;

for $(\$ j=2 ; \$ j<=4 ; \$ j++)\{$

$\$ k=\$ j+3$;

$\$ y=\$ y+\$$ disasters $[\$ i][\$ j] *$ disasters $[\$ i]$

$[\$ k]$;

\}

$\$$ disasters $[\$ i][8]=\$ y$;

echoCHtml::submitButton(\$disasters->isNew Record? 'Create' : 'Save';

\} 
在灾情数据库中, 每一个行政单元对应一条记 录。假设 disasters 数据表中的每条记录的第 1-2 个元 素表示该行政单元名称及其编号, 第 3-5 个元素分别 表示该单元的人口数量、GDP 和相对暴露度, 第 6-8 个元素分别表示上述变量的回归系数。为了提高效率, 使用 foreach()函数按序依此读取 10 条记录为数组格 式并对其进行迭代。使用 for 循环语句实现对式 (2) 的计算。CHtml::submitButton ()在网页上创建了提交 按钮, 将最新灾情评估结果保存至数据库。将上述代 码写入 test.php 文件, 通过第三节所述步骤定时执行, 即可实现在线定时灾情评估功能。

\section{7. 结论与讨论}

仙台框架指出, 全球气候变化的加剧和人类对自 然的利用深度的增加使得自然灾害形势愈发严峻 ${ }^{[9]}$, 我国作为世界上承受自然灾害损失最为严重的国家 之一, 更需大力支持和发展防灾减灾救灾支撑原理和 技术的发展, 科学应对自然灾害。

在应对自然灾害尤其是重大自然灾害发生时, 灾 情信息往往不完备, 严重制约着灾害救助的精准性与 时效性, 为此本文尝试将地理空间信息扩散技术于智 联网理念相结合, 搭建自然灾害灾情快速评估平台, 通过救灾智联网系统实现灾情实时跟踪和自动评估, 从而助力防灾减灾工作, 降低灾后由于救助方案不当 产生的链式不良后果, 保障灾区有效恢复。

为实现救灾智联网的构想, 一方面依赖于能表达 与地理空间相关的非线性关系的算法的发展, 另一方 面则要在现有智联网技术上做新的突破。本文着重从 数据库的设计与管理、模型计算模块与数据库的衔接 以及如何实现在线自动计算这三个方面进行了技术 路径的探讨, 给出了救灾智联网实现的基本框架。 目前我国防灾减灾相关平台数量和业务范围较为有 限, 且偏重灾害的自然属性, 而未涉及救灾应急和恢
复重建等实操层面, 对于灾害管理人员的参考价 值有限。防灾减灾包括但不仅限于灾害自然机理 研究和灾害监测, 在灾害管理实操方面也需要理 论和技术的支撑, 如救助需求评价、有限资源的 配置优化等问题。救灾智联网是在灾害应急管理 平台建设方面的尝试, 希望能从灾情快速评估这 一侧面出发做出有益探索。

\section{参考文献}

[1] Huang C F. Principle of information diffusion. Fuzzy Sets and Systems, 1997, 91(1): 69-90.

[2] 黄崇福.自然灾害风险评价理论与实践. 北 京: 科学出版社, 2005.

[3] 黄崇福.地理空间上的信息扩散及其在风险 分析中的应用. 一带一路背景下的风险分 析及危机反应, 黄崇福, 王成军编. 巴黎: Atlantis 出版社, 2018, pp.1-7.

[4] 黄崇福. 风险分析在线服务的智联网. Journal of Risk Analysis and Crisis Response, 2011, 1(2): 110-117

[5] 黄崇福.用智联网缓解风险分析中的 4 个问题. 自然灾害学报, 2014, 23(2):1-7

[6] 孙雁南.与智联网相融合的地理信息系统在 风险分析中的应用一以四川省三台县永和 堰灌区的脆弱性评价为例. 北京师范大学 硕士学位论文,2018.

[7] 李容. 基于MVC模式的Web应用研究. 软件 导刊, 2010, 09(1):19-21.

[8] Brunsdon C, Fotheringham A S, Charlton M E. Geographically weighted regression: a method for exploring spatial nonstationarity. Geographical Analysis, 28(4): 281-298, 1996

[9] UNGA, Sendai Framework for Disaster Risk Reduction 2015-2030. UN, Sendai, Japan, 2015.http://www.un-isdr.org/we/inform/publi cations/43291 